www.czasopisma.uni.lodz.pl/foe/

2(353) 2021

DOI: https://doi.org/10.18778/0208-6018.353.04

\title{
Małgorzata Graczyk
}

Poznań University of Life Sciences, Faculty of Agronomy and Bioengineering

Department of Mathematical and Statistical Methods, malgorzata.graczyk@up.poznan.pl

\section{Bronisław Ceranka}

Poznań University of Life Sciences, Faculty of Agronomy and Bioengineering

Department of Mathematical and Statistical Methods, bronislaw.ceranka@up.poznan.pl

\section{Selected Remarks on Highly D-efficient Spring Balance Weighing Designs}

\begin{abstract}
Here, we consider a new construction method of determining highly D-efficient spring balance weighing designs in classes in which a D-optimal design does not exist. We give some conditions determining the relations between the parameters of such designs and construction examples.
\end{abstract}

Keywords: D-efficient design, spring balance weighing design

JEL: C02, C18, C90 


\section{Introduction}

Let us consider $\Psi_{n \times p}(0,1)$, a class of $n \times p$ matrices $\mathbf{X}=\left(x_{i j}\right), i=1,2, \ldots, n, j=1,2, . ., p$. Any matrix $\mathbf{X} \in \Psi_{n \times p}(0,1)$ is called a design matrix of the spring balance weighing design. Originally, the name spring balance weighing design pertained to experiments connected with determining unknown weights of objects by the use of balance with one pan which is called a spring balance. Nowadays, such designs are applied in many branches of knowledge including economic survey, see Banerjee (1975), Ceranka and Graczyk (2014). Some aspects of applications of spring balance weighing designs in agriculture are given by Ceranka and Katulska (1987a; 1987b; 1989), and Graczyk (2013). The example of application of such designs in bioengineering is presented in Gawande and Patkar (1999). Various problems related to spring balance weighing designs are presented in the literature. They are focused on the optimality criteria of such designs. The classic works here are Jacroux and Notz (1983), Koukouvinos (1996). Another group of issues is concerned with determining new methods of construction of the design matrices satisfying optimality conditions. The best general references here are Gail and Kiefer (1982), Ceranka and Graczyk (2010; 2012), Katulska and Smaga (2010).

For any matrix $\mathbf{X} \in \Psi_{n \times p}(0,1)$, we consider a linear model:

$$
\mathbf{y}=\mathbf{X w}+\mathbf{e},
$$

where y is an $n \times 1$ random vector of observed measurements. Moreover, $\mathrm{w}$ is a $p \times 1$ vector representing unknown measurements of objects and w is an $n \times 1$ vector of random errors. We make two standing assumptions: it is required that there are no systematic errors, i.e. $E(\mathbf{e})=0_{n}$, and that the errors are uncorrelated and have different variances, i.e. $\operatorname{Var}(\mathbf{e})=\sigma^{2} \mathbf{G}$, where $\sigma>0$ is a known parameter, $\mathrm{G}$ is the $n \times n$ diagonal positive definite matrix of known elements.

For the estimation of the vector of unknown measurements of objects $\mathbf{w}$, we use the normal equation $\mathbf{X}^{\prime} \mathbf{G}^{-1} \mathbf{X} \hat{\mathbf{w}}=\mathbf{X}^{\prime} \mathbf{G}^{-1} \mathbf{y}$. Under the assumption that $\mathbf{G}$ is a known positive definite matrix, $\mathbf{X}^{\prime} \mathbf{G}^{-1} \mathbf{X}$ is nonsingular if and only if $\mathbf{X}$ is of full column rank. In the case when $\mathbf{X}^{\prime} \mathbf{G}^{-1} \mathbf{X}$ is nonsingular, the generalised least squares estimator of $\mathbf{w}$ is given by $\hat{\mathbf{w}}=\left(\mathbf{X}^{\prime} \mathbf{G}^{-1} \mathbf{X}\right)^{-1} \mathbf{X}^{\prime} \mathbf{G}^{-1} \mathbf{y}$ and $\operatorname{Var}(\hat{\mathbf{w}})=$ $\sigma^{2}\left(\mathbf{X}^{\prime} \mathbf{G}^{-1} \mathbf{X}\right)^{-1}$.

The statistical problem considered here is how to determine the estimator of the vector of unknown measurements of objects $\mathbf{w}$ when the observations follow the model (1). Among several questions taken under consideration, the properties of this estimator are under considerations. The characteristic features are determined by the properties of the design. Especially here, it is expected that the product of the variance of the estimators has attained the lowest bound. Hence, the criterion of the D-optimality is considered. The design $\mathbf{X}_{\mathrm{D}}$ is D-optimal in the class 
of the designs $\Psi_{n \times p}(0,1)$ if $\operatorname{det}\left(\mathbf{X}^{\prime} \mathbf{G}^{-1} \mathbf{X}\right)^{-1}=\min \left\{\operatorname{det}\left(\mathbf{X}^{\prime} \mathbf{G}^{-1} \mathbf{X}\right)^{-1}: \mathbf{X} \in \Psi_{n \times p}(0,1)\right\}$

It is known that $\operatorname{det}\left(\mathbf{X}^{\prime} \mathbf{G}^{-1} \mathbf{X}\right)^{-1}$ is minimal if and only if $\operatorname{det}\left(\mathbf{X}^{\prime} \mathbf{G}^{-1} \mathbf{X}\right)$ is maximal. The concept of D-optimality was considered in the books of Raghavarao (1971), Banerjee (1975), as well as Shah and Sinh (1989). Although theoretical studies on providing knowledge to guide the selection of optimal designs are not scarce, we are still unable to determine a regular D-optimal design for any combination of the number of objects and the number of measurements.

In such a case, a highly D-efficient design is considered. For details, we refer the reader to Bulutoglu and Ryan (2009). In Ceranka and Graczyk's (2018) paper, the definition of D-efficiency is given. We indicate a highly D-efficient design when $\mathrm{D}_{\text {eff }}=\left[\frac{\operatorname{det}\left(\mathbf{X}^{\prime} \mathbf{X}\right)}{\operatorname{det}\left(\mathbf{Y}^{\prime} \mathbf{Y}\right)}\right]^{1 / p} \geq 0.95$, where $\mathbf{Y}$ is the matrix of D-optimal spring balance weighing design.

The aim of this paper is to develop new construction methods related to D-optimal and highly D-efficient spring balance weighing designs for which random errors are uncorrelated and have different variances. An attempt has been made here to expand the theory of optimal designs. The aim of this research is to develop the results concerning new methods of determining optimal designs in classes in which they have not been determined in the literature so far.

\section{The main result}

We present the theorem determining the parameters of the highly D-efficient design given in Ceranka and Graczyk (2018; 2019).

Theorem 2.1. Let $p$ be even. In any non-singular spring balance weighing design $\mathbf{X} \in \Psi_{n \times p}(0,1)$ having $0.5 p$ ones in each row and with the variance matrix of errors $\sigma^{2} \mathbf{I}_{n}, \operatorname{det}\left(\mathbf{X}^{\prime} \mathbf{X}\right) \leq(p-1)\left(\frac{n p}{4(p-1)}\right)^{p}$, an upper bound is attained if and only if $\mathbf{X}^{\prime} \mathbf{X}=\frac{n}{4(p-1)}\left(p \mathbf{I}_{p}+(p-2) \mathbf{1}_{p} \mathbf{1}_{p}^{\prime}\right)$, where $0.25 n(p-2)(p-1)^{-1}$ and $0.25 n p(p-1)^{-1}$ are integers.

The design $\mathbf{X} \in \Psi_{n \times p}(0,1)$ having the form given in Theorem 2.1 is considered as highly D-efficient. 


\subsection{Addition of one measurement}

Let $\mathbf{X}_{1} \in \Psi_{(n-1) \times p}(0,1)$ be the design of the highly D-efficient spring balance weighing design. Now, let us consider the design $\mathbf{X} \in \Psi_{n \times p}(0,1)$ in the form:

$$
\mathbf{X}=\left[\begin{array}{c}
\mathbf{X}_{1} \\
\mathbf{x}_{1}^{\prime}
\end{array}\right]
$$

where $\mathbf{x}_{1}$ is any $p \times 1$ vector of elements 1 or $0, \mathbf{x}_{1}^{\prime} \mathbf{1}_{p}=t_{1}, 1 \leq t_{1} \leq p$. So, the variance matrix of errors is given as:

$$
\mathbf{G}=\left[\begin{array}{cc}
\mathbf{I}_{n-1} & \mathbf{0}_{n-1} \\
\mathbf{0}_{n-1}^{\prime} & g_{1}^{-1}
\end{array}\right] .
$$

Furthermore, we study the function $\operatorname{det}\left(\mathbf{X}^{\prime} \mathbf{G}^{-1} \mathbf{X}\right)$.

Because $\mathbf{X}_{1}^{\prime} \mathbf{X}_{1}=\frac{n-1}{4(p-1)}\left(p \mathbf{I}_{p}+(p-2) \mathbf{1}_{p} \mathbf{1}_{p}^{\prime}\right)$, then $\operatorname{det}\left(\mathbf{X}_{1}^{\prime} \mathbf{X}_{1}\right) \leq(p-1)\left(\frac{(n-1) p}{4(p-1)}\right)^{p}$ and the determinant is maximal if and only if $\frac{(n-1) p}{4(p-1)}$ and $\frac{(n-1)(p-2)}{4(p-1)}$ are integers.

Thus for the design matrix $\mathbf{X} \in \Psi_{n \times p}(0,1)$, we have

$$
\operatorname{det}\left(\mathbf{X}^{\prime} \mathbf{G}^{-1} \mathbf{X}\right) \leq(p-1)\left(\frac{(n-1) p}{4(p-1)}\right)^{p}\left(1+g_{1} \mathbf{x}_{1}^{\prime}\left(\mathbf{X}_{1}^{\prime} \mathbf{X}_{1}\right)^{-1} \mathbf{x}_{1}\right) .
$$

Owing to the fact that $\left(\mathbf{X}_{1}^{\prime} \mathbf{X}_{1}\right)^{-1}=\frac{4(p-1)}{(n-1) p}\left(\mathbf{I}_{p}-\frac{p-2}{p(p-1)} \mathbf{1}_{p} \mathbf{1}_{p}^{\prime}\right)$, we obtain:

$$
\begin{gathered}
\operatorname{det}\left(\mathbf{X}^{\prime} \mathbf{G}^{-1} \mathbf{X}\right) \leq(p-1)\left(\frac{(n-1) p}{4(p-1)}\right)^{p}\left(1+g_{1} \mathbf{x}_{1} \frac{4(p-1)}{(n-1) p}\left(\mathbf{I}_{p}-\frac{p-2}{p(p-1)} \mathbf{1}_{p} \mathbf{1}_{p}^{\prime}\right) \mathbf{x}_{1}\right)= \\
(p-1)\left(\frac{(n-1) p}{4(p-1)}\right)^{p}\left(1+\frac{4(p-1) g_{1}}{(n-1) p}\left(\mathbf{x}_{1}^{\prime} \mathbf{x}_{1}-\frac{p-2}{p(p-1)} \mathbf{x}_{1}^{\prime} \mathbf{1}_{p} \mathbf{1}_{p}^{\prime} \mathbf{x}_{1}\right)\right) .
\end{gathered}
$$

In order to maximise the expression to the right of the inequality sign, we observe that $t_{1}-\frac{p-2}{p(p-1)} t_{1}^{2} \leq \frac{p^{3}+8}{4 p(p-1)}$ and the equality in the above-presented inequality holds if and only if $t_{1}=\frac{p+2}{2}$. Hence, 


$$
\begin{aligned}
\operatorname{det}\left(\mathbf{X}^{\prime} \mathbf{G}^{-1} \mathbf{X}\right) \leq & (p-1)\left(\frac{(n-1) p}{4(p-1)}\right)^{p}\left(1+\frac{4(p-1) g_{1}}{(n-1) p} \cdot \frac{p^{3}+8}{4 p(p-1)}\right)= \\
& (p-1)\left(\frac{(n-1) p}{4(p-1)}\right)^{p}\left(1+\frac{g_{1}\left(p^{3}+8\right)}{(n-1) p^{2}}\right) .
\end{aligned}
$$

The above-presented equality is fulfilled if and only if $t_{1}=\frac{p+2}{2}$ and $\frac{(n-1) p}{4(p-1)}$ and $\frac{(n-1)(p-2)}{4(p-1)}$ are integer numbers.

Theorem 2.2. Let $p$ be even. Any spring balance weighing design $\mathbf{X} \in \Psi_{n \times p}(0,1)$ in the form (2.1) with the variance matrix of errors in the form (2.2) is highly D-efficient in the class $\Psi_{n \times p}(0,1)$ if and only if $t_{1}=\frac{p+2}{2}$ and $\frac{(n-1) p}{4(p-1)}$ and $\frac{(n-1)(p-2)}{4(p-1)}$ are integer numbers.

Definition 2.1. Any spring balance weighing design $\mathbf{X} \in \Psi_{n \times p}(0,1)$ in the form (2.1) with the variance matrix of errors in the form (2.2) is highly D-efficient if:

$$
\operatorname{det}\left(\mathbf{X}^{\prime} \mathbf{G}^{-1} \mathbf{X}\right) \leq(p-1)\left(\frac{(n-1) p}{4(p-1)}\right)^{p}\left(1+\frac{g_{1}\left(p^{3}+8\right)}{(n-1) p^{2}}\right)
$$

Example 2.1. Let us consider the variance matrix of errors $\sigma^{2} \mathbf{G}$, where $\mathbf{G}^{-1}=\left[\begin{array}{cc}\mathbf{I}_{6} & \mathbf{0}_{6} \\ \mathbf{0}_{6}^{\prime} & 5\end{array}\right]$. We determine a highly D-efficient design in the class $\mathbf{X} \in \Psi_{7 \times 4}(0,1)$. So, take the highly D-efficient spring balance weighing design $\mathbf{X}_{1} \in \Psi_{6 \times 4}(0,1)$ in the form $\quad \mathbf{X}_{1}=\left[\begin{array}{llll}1 & 1 & 0 & 0 \\ 1 & 0 & 1 & 0 \\ 1 & 0 & 0 & 1 \\ 0 & 1 & 1 & 0 \\ 0 & 1 & 0 & 1 \\ 0 & 0 & 1 & 1\end{array}\right]$. If $\quad \mathbf{x}_{1}^{\prime}=\left[\begin{array}{llll}1 & 1 & 1 & 0\end{array}\right]$. So,

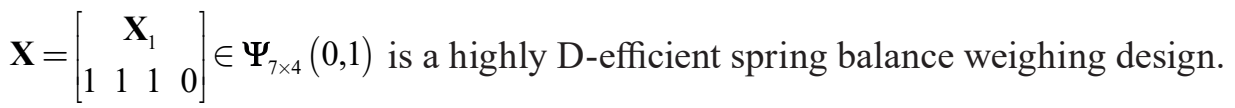

Example 2.2. Let us consider the variance matrix of errors $\sigma^{2} \mathbf{G}$, where $\mathbf{G}^{-1}=\left[\begin{array}{cc}\mathbf{I}_{10} & \mathbf{0}_{10} \\ \mathbf{0}_{10}^{\prime} & 5\end{array}\right]$. We determine a highly D-efficient design in the class $\mathbf{X} \in \Psi_{11 \times 6}(0,1)$. So, take the highly D-efficient spring balance weighing design 
$\mathbf{X}_{1} \in \Psi_{10 \times 6}(0,1)$ in the form $\mathbf{X}_{1}=\left[\begin{array}{llllll}1 & 1 & 0 & 0 & 1 & 0 \\ 1 & 1 & 0 & 0 & 0 & 1 \\ 1 & 0 & 1 & 1 & 0 & 0 \\ 1 & 0 & 1 & 0 & 0 & 1 \\ 1 & 0 & 0 & 1 & 1 & 0 \\ 0 & 1 & 1 & 1 & 0 & 0 \\ 0 & 1 & 1 & 0 & 1 & 0 \\ 0 & 1 & 0 & 1 & 0 & 1 \\ 0 & 0 & 1 & 0 & 1 & 1 \\ 0 & 0 & 0 & 1 & 1 & 1\end{array}\right]$. If $\mathbf{x}_{1}^{\prime}=\left[\begin{array}{lllll}1 & 1 & 1 & 1 & 0\end{array}\right]$, then

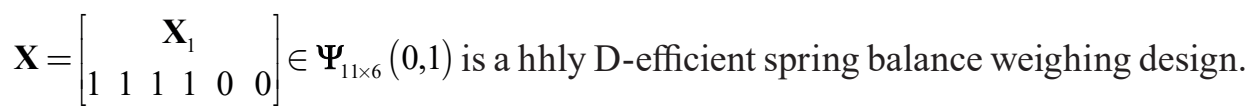

\subsection{Addition of two measurements}

Let $\mathbf{X}_{1} \in \Psi_{(n-2) \times p}(0,1)$ be the design of the highly D-efficient spring balance weighing design. Now, let us consider the design $\mathbf{X} \in \Psi_{n \times p}(0,1)$ in the form:

$$
\mathbf{X}=\left[\begin{array}{l}
\mathbf{X}_{1} \\
\mathbf{x}_{1}^{\prime} \\
\mathbf{x}_{2}^{\prime}
\end{array}\right]
$$

where $\mathbf{x}_{h}$ is a $p \times 1$ vector of elements 1 or $0, \mathbf{x}_{h}^{\prime} \mathbf{1}_{p}=t_{h}, h=1,2, \mathbf{x}_{1}^{\prime} \mathbf{x}_{2}=u_{12}, 1 \leq t_{h} \leq p$. So, the variance matrix of errors is given as:

$$
\mathbf{G}=\left[\begin{array}{ccc}
\mathbf{I}_{n-2} & \mathbf{0}_{n-2} & \mathbf{0}_{n-2} \\
\mathbf{0}_{n-2}^{\prime} & g_{1}^{-1} & 0 \\
\mathbf{0}_{n-2}^{\prime} & 0 & g_{2}^{-1}
\end{array}\right]
$$

Furthermore, we study the function $\operatorname{det}\left(\mathbf{X}^{\prime} \mathbf{G}^{-1} \mathbf{X}\right)$.

$$
\text { Because } \mathbf{X}_{1}^{\prime} \mathbf{X}_{1}=\frac{n-2}{4(p-1)}\left(p \mathbf{I}_{p}+(p-2) \mathbf{1}_{p} \mathbf{1}_{p}^{\prime}\right), \text { then } \operatorname{det}\left(\mathbf{X}_{1}^{\prime} \mathbf{X}_{1}\right) \leq(p-1)\left(\frac{(n-2) p}{4(p-1)}\right)^{p}
$$

and the maximum value is attained if and only if $\frac{(n-2) p}{4(p-1)}$ and $\frac{(n-2)(p-2)}{4(p-1)}$ are integers. Thus, $\operatorname{det}\left(\mathbf{X}^{\prime} \mathbf{G}^{-1} \mathbf{X}\right) \leq(p-1)\left(\frac{(n-2) p}{4(p-1)}\right)^{p} \operatorname{det}\left(\mathbf{I}_{2}+\left[\begin{array}{l}g_{1} \mathbf{x}_{1}^{\prime} \\ g_{2} \mathbf{x}_{2}^{\prime}\end{array}\right]\left(\mathbf{X}_{1}^{\prime} \mathbf{X}_{1}\right)^{-1} \mathbf{x}_{1} \mathbf{x}_{2}\right)$. 
Since $\left(\mathbf{X}_{1}^{\prime} \mathbf{X}_{1}\right)^{-1}=\frac{4(p-1)}{(n-2) p}\left(\mathbf{I}_{p}-\frac{p-2}{p(p-1)} \mathbf{1}_{p} \mathbf{1}_{p}^{\prime}\right)$, then we have $\operatorname{det}\left(\mathbf{X}^{\prime} \mathbf{G}^{-1} \mathbf{X}\right) \leq(p-1)\left(\frac{(n-2) p}{4(p-1)}\right)^{p} \operatorname{det}\left(\mathbf{T}_{2}\right)$, here:

$$
\begin{gathered}
\mathbf{T}_{2}=\mathbf{I}_{2}+\left[\begin{array}{l}
g_{1} \mathbf{x}_{1}^{\prime} \\
g_{2} \mathbf{x}_{2}^{\prime}
\end{array}\right] \frac{4(p-1)}{(n-2) p}\left(\mathbf{I}_{p}-\frac{p-2}{p(p-1)} 1_{p} 1_{p}^{\prime}\right)\left[\mathbf{x}_{1} \mathbf{x}_{2}\right] \\
\operatorname{det}\left(\mathbf{T}_{2}\right)=\left[1+\frac{4(p-1) g_{1}}{(n-2) p}\left(t_{1}-\frac{p-2}{p(p-1)} t_{1}^{2}\right)\right] \cdot\left[1+\frac{4(p-1) g_{2}}{(n-2) p}\left(t_{2}-\frac{p-2}{p(p-1)} t_{2}^{2}\right)\right]- \\
\left(\frac{4(p-1)}{(n-2) p}\right)^{2} g_{1} g_{2}\left(u_{12}-\frac{p-2}{p(p-1)} t_{1} t_{2}\right)^{2} .
\end{gathered}
$$

$\operatorname{det}\left(\mathbf{T}_{2}\right)$ takes the maximum value if $t_{h}-\frac{p-2}{p(p-1)} t_{h}^{2} \leq \frac{p^{3}+8}{4 p(p-1)}$ and the equality is fulfilled if and only if $t_{h}=\frac{p+2}{2}, h=1,2$.

Hence,

$$
\begin{aligned}
& \operatorname{det}\left(\mathbf{T}_{2}\right) \leq {\left[1+\frac{4(p-1) g_{1}}{(n-2) p} \frac{p^{3}+8}{4 p(p-1)}\right] \cdot\left[1+\frac{4(p-1) g_{2}}{(n-2) p} \frac{p^{3}+8}{4 p(p-1)}\right]-} \\
&\left(\frac{4(p-1)}{(n-2) p}\right)^{2} g_{1} g_{2}\left(u_{12}-\frac{(p-2)(p+2)^{2}}{4 p(p-1)}\right)^{2}= \\
&\left(1+\frac{g_{1}\left(p^{3}+8\right)}{(n-2) p^{2}}\right)\left(1+\frac{g_{2}\left(p^{3}+8\right)}{(n-2) p^{2}}\right)-\left(\frac{4(p-1)}{(n-2) p}\right)^{2} g_{1} g_{2}\left(u_{12}-\frac{(p-2)(p+2)^{2}}{4 p(p-1)}\right)^{2} .
\end{aligned}
$$

$u_{12}$ is an integer number. Now, we consider two cases: $p \equiv 0 \bmod 4$ and $p+2 \equiv 0 \bmod 4$.

If $p \equiv 0 \bmod 4$, then $u_{12}=0.25(p+4)$ and

$\left(u_{12}-\frac{(p-2)(p+2)^{2}}{4 p(p-1)}\right)^{2}=\left(\frac{p+4}{4}-\frac{(p-2)(p+2)^{2}}{4 p(p-1)}\right)^{2}=\left(\frac{p^{2}+8}{4 p(p-1)}\right)^{2}$.

In this situation,

$\operatorname{det}\left(\mathbf{T}_{2}\right) \leq\left(1+\frac{g_{1}\left(p^{3}+8\right)}{(n-2) p^{2}}\right) \cdot\left(1+\frac{g_{2}\left(p^{3}+8\right)}{(n-2) p^{2}}\right)-\left(\frac{4(p-1)}{(n-2) p}\right)^{2} g_{1} g_{2}\left(\frac{p^{2}+8}{4 p(p-1)}\right)^{2}$ and 


$$
\begin{gathered}
\operatorname{det}\left(\mathbf{X}^{\prime} \mathbf{G}^{-1} \mathbf{X}\right) \leq(p-1)\left(\frac{(n-2) p}{4(p-1)}\right)^{p} \\
\left\{\left(1+\frac{g_{1}\left(p^{3}+8\right)}{(n-2) p^{2}}\right) \cdot\left(1+\frac{g_{2}\left(p^{3}+8\right)}{(n-2) p^{2}}\right)-g_{1} g_{2}\left(\frac{p^{2}+8}{p^{2}(n-2)}\right)^{2}\right\}
\end{gathered}
$$

Theorem 2.3. Let $p \equiv 0 \bmod 4$. Any spring balance weighing design $\mathbf{X} \in \Psi_{n \times p}(0,1)$ in the form (2.3) with the variance matrix of errors in the form (2.4) is highly D-efficient in the class $\Psi_{n \times p}(0,1)$ if and only if $t_{h}=\frac{p+2}{2}$ and $\frac{(n-1) p}{4(p-1)}$ and $\frac{(n-2)(p-2)}{4(p-1)}$ are integer numbers, $u_{12}=0.25(p+4), h=1,2$.

Definition 2.2. Let $p \equiv 0 \bmod 4$. Any spring balance weighing design $\mathbf{X}$ in the form (2.3) with the variance matrix of errors in the form (2.4) is highly D-efficient if

$$
\begin{gathered}
\operatorname{det}\left(\mathbf{X}^{\prime} \mathbf{G}^{-1} \mathbf{X}\right)=(p-1)\left(\frac{(n-2) p}{4(p-1)}\right)^{p} \\
\left\{\left(1+\frac{g_{1}\left(p^{3}+8\right)}{(n-2) p^{2}}\right) \cdot\left(1+\frac{g_{2}\left(p^{3}+8\right)}{(n-2) p^{2}}\right)-g_{1} g_{2}\left(\frac{p^{2}+8}{p^{2}(n-2)}\right)^{2}\right\}
\end{gathered}
$$

Now, we turn to the case $p+2 \equiv 0 \bmod 4$.

If $p+2 \equiv 0 \bmod 4$, then $u_{12}=0.25(p+2)$ and

$$
\left(u_{12}-\frac{(p-2)(p+2)^{2}}{4 p(p-1)}\right)^{2}=\left(\frac{p+2}{4}-\frac{(p-2)(p+2)^{2}}{4 p(p-1)}\right)^{2}=\left(\frac{(p+2)(p-4)}{4 p(p-1)}\right)^{2} \text {. }
$$

In this case,

$$
\operatorname{det}\left(\mathbf{T}_{2}\right) \leq\left(1+\frac{g_{1}\left(p^{3}+8\right)}{(n-2) p^{2}}\right) \cdot\left(1+\frac{g_{2}\left(p^{3}+8\right)}{(n-2) p^{2}}\right)-\left(\frac{4(p-1)}{(n-2) p}\right)^{2} g_{1} g_{2}\left(\frac{(p+2)(p-4)}{4 p(p-1)}\right)^{2}
$$

and

$$
\begin{gathered}
\operatorname{det}\left(\mathbf{X}^{\prime} \mathbf{G}^{-1} \mathbf{X}\right) \leq(p-1)\left(\frac{(n-2) p}{4(p-1)}\right)^{p} \\
\left\{\left(1+\frac{g_{1}\left(p^{3}+8\right)}{(n-2) p^{2}}\right) \cdot\left(1+\frac{g_{2}\left(p^{3}+8\right)}{(n-2) p^{2}}\right)-g_{1} g_{2}\left(\frac{(p+2)(p-4)}{p^{2}(n-2)}\right)^{2}\right\} .
\end{gathered}
$$

Theorem 2.4. Let $p+2 \equiv 0 \bmod 4$. Any spring balance weighing design $\mathbf{X} \in \Psi_{n \times p}(0,1)$ in the form (2.3) with the variance matrix of errors in the form (2.4) 
is highly D-efficient in the class $\Psi_{n \times p}(0,1)$ if and only if $t_{h}=\frac{p+2}{2}$ and $\frac{(n-2) p}{4(p-1)}$ and $\frac{(n-2)(p-2)}{4(p-1)}$ are integer numbers, $u_{12}=0.25(p+2), h=1,2$.

Definition 2.3. Let $p+2 \equiv 0 \bmod 4$. Any spring balance weighing design $\mathbf{X}$ in the form (2.3) with the variance matrix of errors in the form (2.4) is highly D-efficient if

$$
\begin{gathered}
\operatorname{det}\left(\mathbf{X}^{\prime} \mathbf{G}^{-1} \mathbf{X}\right)=(p-1)\left(\frac{(n-2) p}{4(p-1)}\right)^{p} \\
\left\{\left(1+\frac{g_{1}\left(p^{3}+8\right)}{(n-2) p^{2}}\right) \cdot\left(1+\frac{g_{2}\left(p^{3}+8\right)}{(n-2) p^{2}}\right)-g_{1} g_{2}\left(\frac{(p+2)(p-4)}{p^{2}(n-2)}\right)^{2}\right\}
\end{gathered}
$$

Example 2.3. Let us consider the variance matrix of errors

$$
\sigma^{2} \mathbf{G}, \text { where } \mathbf{G}^{-1}=\left[\begin{array}{ccc}
\mathbf{I}_{6} & \mathbf{0}_{6} & \mathbf{0}_{6} \\
\mathbf{0}_{6}^{\prime} & 5 & 0 \\
\mathbf{0}_{6}^{\prime} & 0 & 3
\end{array}\right] .
$$

We determine a highly D-efficient design in the class $\mathbf{X} \in \Psi_{8 \times 4}(0,1)$. So, take the highly D-efficient spring balance weighing design $\mathbf{X}_{1} \in \Psi_{6 \times 4}(0,1)$ in the form

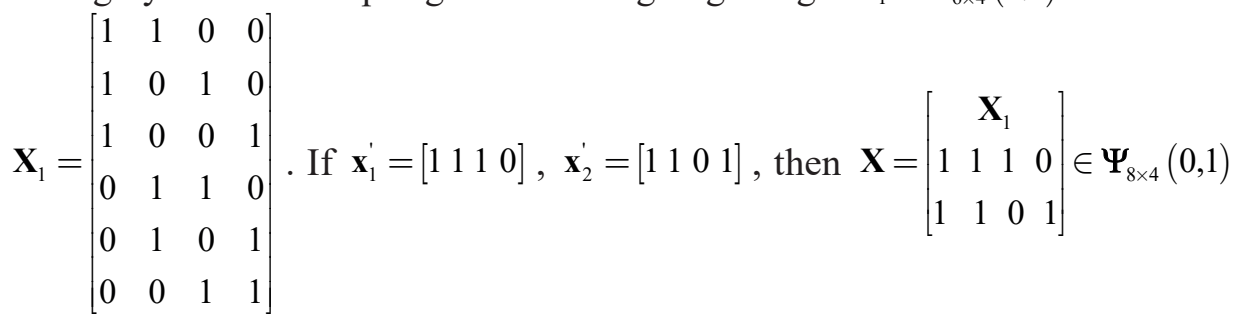
is a highly D-efficient spring balance weighing design.

Example 2.4. Let us consider the variance matrix of errors $\sigma^{2} \mathbf{G}$, where $\mathbf{G}^{-1}=\left[\begin{array}{ccc}\mathbf{I}_{10} & \mathbf{0}_{10} & \mathbf{0}_{10} \\ \mathbf{0}_{10}^{\prime} & 5 & 0 \\ \mathbf{0}_{10}^{\prime} & 0 & 3\end{array}\right]$. We determine a highly D-efficient design in the class $\mathbf{X} \in \Psi_{12 \times 6}(0,1)$. So, take the highly D-efficient spring balance weighing design 


$$
\mathbf{X}_{1} \in \Psi_{10 \times 6}(0,1) \text { in the form } \mathbf{X}_{1}=\left[\begin{array}{cccccc}
1 & 1 & 0 & 0 & 1 & 0 \\
1 & 1 & 0 & 0 & 0 & 1 \\
1 & 0 & 1 & 1 & 0 & 0 \\
1 & 0 & 1 & 0 & 0 & 1 \\
1 & 0 & 0 & 1 & 1 & 0 \\
0 & 1 & 1 & 1 & 0 & 0 \\
0 & 1 & 1 & 0 & 1 & 0 \\
0 & 1 & 0 & 1 & 0 & 1 \\
0 & 0 & 1 & 0 & 1 & 1 \\
0 & 0 & 0 & 1 & 1 & 1
\end{array}\right] . \text { If } \mathbf{x}_{1}^{\prime}=\left[\begin{array}{lllll}
1 & 1 & 1 & 1 & 0
\end{array}\right],
$$

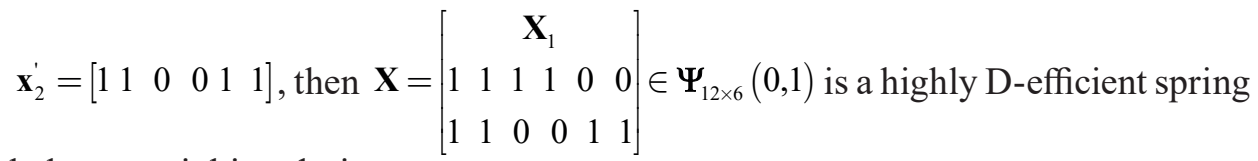
balance weighing design.

\subsection{Addition of three measurements}

Let $\mathbf{X}_{1} \in \Psi_{(n-3) \times p}(0,1)$ be the design of the highly D-efficient spring balance weighing design. Now, let us consider the design $\mathbf{X} \in \Psi_{n \times p}(0,1)$ in the form:

$$
\mathbf{X}=\left[\begin{array}{l}
\mathbf{X}_{1} \\
\mathbf{x}_{1}^{\prime} \\
\mathbf{x}_{2}^{\prime} \\
\mathbf{x}_{3}^{\prime}
\end{array}\right],
$$

where $\mathbf{x}_{h}^{\prime} \mathbf{1}_{p}=t_{h}, \mathbf{x}_{h}^{\prime} \mathbf{x}_{s}=u_{h s}, h, s=1,2,3, h \neq s$. So, the variance matrix of errors is given as:

$$
\mathbf{G}=\left[\begin{array}{cccc}
\mathbf{I}_{n-3} & \mathbf{0}_{n-3} & \mathbf{0}_{n-3} & \mathbf{0}_{n-3} \\
\mathbf{0}_{n-3}^{\prime} & g_{1}^{-1} & 0 & 0 \\
\mathbf{0}_{n-3}^{\prime} & 0 & g_{2}^{-1} & 0 \\
\mathbf{0}_{n-3}^{\prime} & 0 & 0 & g_{3}^{-1}
\end{array}\right] .
$$


Westudythefunction $\operatorname{det}\left(\times^{\prime} \mathbf{G}^{-1} \times\right)$. Because $\mathbf{X}_{1}^{\prime} \mathbf{X}_{1}=\frac{n-3}{4(p-1)}\left(p \mathbf{I}_{p}+(p-2) \mathbf{1}_{p} \mathbf{1}_{p}^{\prime}\right)$, then $\operatorname{det}\left(\mathbf{X}_{1}^{\prime} \mathbf{X}_{1}\right) \leq(p-1)\left(\frac{(n-3) p}{4(p-1)}\right)^{p}$ and the maximum is attained if and only if $\frac{(n-3) p}{4(p-1)}$ and $\frac{(n-3)(p-2)}{4(p-1)}$ are integers. Consequently,

$$
\operatorname{det}\left(\mathbf{X}^{\prime} \mathbf{G}^{-1} \mathbf{X}\right) \leq(p-1)\left(\frac{(n-3) p}{4(p-1)}\right)^{p} \operatorname{det}\left(\mathbf{I}_{3}+\left[\begin{array}{l}
g_{1} \mathbf{x}_{1}^{\prime} \\
g_{2} \mathbf{x}_{2}^{\prime} \\
g_{2} \mathbf{x}_{3}^{\prime}
\end{array}\right]\left(\mathbf{X}_{1}^{\prime} \mathbf{X}_{1}\right)^{-1}\left[\mathbf{x}_{1} \mathbf{x}_{2} \mathbf{x}_{3}\right]\right)
$$

Since $\left(\mathbf{X}_{1}^{\prime} \mathbf{X}_{1}\right)^{-1}=\frac{4(p-1)}{(n-3) p}\left(\mathbf{I}_{p}-\frac{p-2}{p(p-1)} \mathbf{1}_{p} \mathbf{1}_{p}^{\prime}\right)$, then

$\operatorname{det}\left(\mathbf{X}^{\prime} \mathbf{G}^{-1} \mathbf{X}\right) \leq(p-1)\left(\frac{(n-3) p}{4(p-1)}\right)^{p} \operatorname{det}\left(\mathbf{T}_{3}\right)$, where

$\mathbf{T}_{3}=\mathbf{I}_{3}+\left[\begin{array}{l}g_{1} \mathbf{x}_{1}^{\prime} \\ g_{2} \mathbf{x}_{2}^{\prime} \\ g_{2} \mathbf{x}_{3}^{\prime}\end{array}\right] \frac{4(p-1)}{(n-3) p}\left(\mathbf{I}_{p}-\frac{p-2}{p(p-1)} \mathbf{1}_{p} \mathbf{1}_{p}^{\prime}\right)\left[\mathbf{x}_{1} \mathbf{x}_{2} \mathbf{x}_{3}\right]$. By extension, we obtain $\operatorname{det}\left(\mathbf{T}_{3}\right)=\mathrm{A}_{1}+2 \mathrm{~B}_{1} \mathrm{C}_{1}-\mathrm{D}_{1}$, where $\mathrm{A}_{1}=\prod_{h=1}^{3}\left(1+\frac{4(p-1) g_{h}}{(n-3) p}\left(t_{h}-\frac{p-2}{p(p-1)} t_{h}^{2}\right)\right)$, $\mathrm{B}_{1}=\prod_{h=1}^{3} \frac{4(p-1) g_{h}}{(n-3) p}, \mathrm{C}_{1}=\prod_{h, s}\left(u_{h s}-\frac{p-2}{p(p-1)} t_{h} t_{s}\right), h, s=1,2,3, h \neq s$, $\mathrm{D}_{1}=\sum_{h, s, z}\left(\frac{4(p-1) g_{h}}{(n-3) p}\right)^{2}\left(u_{h s}-\frac{p-2}{p(p-1)} t_{h} t_{s}\right)^{2}\left(1+\frac{4(p-1) g_{z}}{(n-3) p}\left(t_{z}-\frac{p-2}{p(p-1)} t_{z}^{2}\right)\right)$, $h, s, z=1,2,3, h \neq s \neq z$. Because $t_{h}-\frac{p-2}{p(p-1)} t_{h}^{2} \leq \frac{p^{3}+8}{4 p(p-1)}$, then the equality is fulfilled if and only if $t_{h}=\frac{p+2}{2}, h=1,2,3$. Hence, $\operatorname{det}\left(\mathbf{T}_{3}\right) \leq \mathrm{A}_{2}+2 \mathrm{~B}_{2} \mathrm{C}_{2}-\mathrm{D}_{2}$, where $\mathrm{A}_{2}=\prod_{h=1}^{3}\left(1+\frac{4(p-1) g_{h}}{(n-3) p} \frac{p^{3}+8}{4 p(p-1)}\right), \mathrm{B}_{2}=\left(\frac{4(p-1)}{(n-3) p}\right)^{3} \prod_{h=1}^{3} g_{h}$, 
$\mathrm{C}_{2}=\prod_{h, s}\left(u_{h s}-\frac{(p-2)(p+2)^{2}}{4 p(p-1)}\right)$,

$\mathrm{D}_{2}=\left(\frac{4(p-1)}{(n-3) p}\right)^{2} \sum_{h, s, z} g_{h} g_{s}\left(u_{h s}-\frac{(p-2)(p+2)^{2}}{4 p(p-1)}\right)^{2}\left(1+\frac{4(p-1) g_{z}}{(n-3) p}\left(\frac{p^{3}+8}{4 p(p-1)}\right)\right)$,

$h, s, z=1,2,3, h \neq s \neq z$. After calculations, we have:

$$
\begin{gathered}
\operatorname{det}\left(\mathbf{T}_{3}\right)=\mathrm{A}_{3}+2 \mathrm{~B}_{2} \mathrm{C}_{2}-\mathrm{D}_{3} \\
\mathrm{~A}_{3}=\prod_{h=1}^{3}\left(1+\frac{g_{h}\left(p^{3}+8\right)}{(n-3) p^{2}}\right), \\
\mathrm{D}_{3}=\left(\frac{4(p-1)}{(n-3) p}\right)^{2} \sum_{h, s, z} g_{h} g_{s}\left(u_{h s}-\frac{(p-2)(p+2)^{2}}{4 p(p-1)}\right)^{2}\left(1+\frac{g_{z}\left(p^{3}+8\right)}{(n-3) p^{2}}\right) .
\end{gathered}
$$

In order to maximise $\operatorname{det}\left(\mathbf{T}_{3}\right)$, we need to calculate a maximum for integer number $u_{h s}$.

Now, we consider two cases: $p \equiv 0 \bmod 4$ and $p+2 \equiv 0 \bmod 4$.

If $p \equiv 0 \bmod 4$, then minimum value of $u_{h s}-\frac{(p-2)(p+2)^{2}}{4 p(p-1)}$ equals

$\left(\frac{p^{2}+8}{4 p(p-1)}\right)^{2}$ for $u_{h s}=0.25(p+4)$. In this case, $\operatorname{det}\left(\mathbf{T}_{3}\right) \leq \mathrm{A}_{3}+2 \mathrm{~B}_{2} \mathrm{C}_{4}-\mathrm{D}_{4}$, where $\mathrm{C}_{4}=\left(\frac{p^{2}+8}{4 p(p-1)}\right)^{3}, \mathrm{D}_{4}=\left(\frac{p^{2}+8}{(n-3) p^{2}}\right)^{2} \sum_{h, s, z} g_{h} g_{s}\left(1+\frac{g_{z}\left(p^{3}+8\right)}{(n-3) p^{2}}\right)$ and $\operatorname{det}\left(\mathbf{X}^{\prime} \mathbf{G}^{-1} \mathbf{X}\right) \leq(p-1)\left(\frac{(n-3) p}{4(p-1)}\right)^{p}\left\{\mathrm{~A}_{4}+2 \mathrm{~B}_{2} \mathrm{C}_{4}-\mathrm{D}_{4}\right\}$. So, we can formulate the following theorem.

Theorem 2.5. Let $p \equiv 0 \bmod 4$. Any spring balance weighing design $\mathbf{X} \in \Psi_{n \times p}(0,1)$ in the form (2.5) with the variance matrix of errors in the form (2.6) 
is highly D-efficient in the class $\Psi_{n \times p}(0,1)$ if and only if $t_{h}=\frac{p+2}{2}$ and $\frac{(n-3) p}{4(p-1)}$ and $\frac{(n-3)(p-2)}{4(p-1)}$ are integer numbers, $u_{h s}=0.25(p+4), h=1,2,3$.

Definition 2.4. Let $p \equiv 0 \bmod 4$. Any spring balance weighing design $\mathbf{X} \in \Psi_{n \times p}(0,1)$ in the form (2.5) with the variance matrix of errors in the form (2.6) is highly D-efficient if;

$$
\begin{gathered}
\operatorname{det}\left(\mathbf{X}^{\prime} \mathbf{G}^{-1} \mathbf{X}\right)=(p-1)\left(\frac{(n-3) p}{4(p-1)}\right)^{p} \\
\left\{\begin{array}{l}
\prod_{h=1}^{3}\left(1+\frac{g_{h}\left(p^{3}+8\right)}{(n-3) p^{2}}\right)+2\left(\frac{p^{2}+8}{(n-3) p^{2}}\right)^{3} \prod_{h=1}^{3} g_{h}- \\
\left(\frac{p^{2}+8}{(n-3) p^{2}}\right)^{2} \sum_{h, s, z} g_{h} g_{s}\left(1+\frac{g_{z}\left(p^{3}+8\right)}{(n-3) p^{2}}\right)
\end{array}\right\} .
\end{gathered}
$$

Now, we turn to the case $p+2 \equiv 0 \bmod 4$.

If $p+2 \equiv 0 \bmod 4$, then $u_{12}=0.25(p+2)$ and

$$
u_{12}-\frac{(p-2)(p+2)^{2}}{4 p(p-1)}=\frac{p+2}{4}-\frac{(p-2)(p+2)^{2}}{4 p(p-1)}=-\frac{(p+2)(p-4)}{4 p(p-1)} .
$$

In this case, $\operatorname{det}\left(\mathbf{T}_{3}\right) \leq \mathrm{A}_{3}+2 \mathrm{~B}_{2} \mathrm{C}_{5}-\mathrm{D}_{5}$, where $\mathrm{C}_{5}=\left(\frac{(p+2)(p-4)}{4 p(p-1)}\right)^{3}$,

$$
\begin{gathered}
\mathrm{D}_{5}=\left(\frac{(p+2)(p-4)}{(n-3) p^{2}}\right)^{2} \sum_{h, s, z} g_{h} g_{s}\left(1+\frac{g_{z}\left(p^{3}+8\right)}{(n-3) p^{2}}\right) \text { and } \\
\operatorname{det}\left(\mathbf{X}^{\prime} \mathbf{G}^{-1} \mathbf{X}\right) \leq(p-1)\left(\frac{(n-3) p}{4(p-1)}\right)^{p}\left\{\mathrm{~A}_{5}+2 \mathrm{~B}_{5} \mathrm{C}_{5}-\mathrm{D}_{5}\right\} .
\end{gathered}
$$

Theorem 2.6. Let $p+2 \equiv 0 \bmod 4$. Any spring balance weighing design $\mathbf{X} \in \Psi_{n \times p}(0,1)$ in the form (2.5) with the variance matrix of errors in the form (2.6) is highly D-efficient if and only if $t_{h}=\frac{p+2}{2}$ and $\frac{(n-3) p}{4(p-1)}$ and $\frac{(n-3)(p-2)}{4(p-1)}$ are integer numbers, $u_{12}=0.25(p+2), h=1,2,3$.

Definition 2.5. Any spring balance weighing design $\mathbf{X} \in \Psi_{n \times p}(0,1)$ in the form (2.53) with the variance matrix of errors in the form (2.6) is highly D-efficient if: 


$$
\begin{gathered}
\operatorname{det}\left(\mathbf{X}^{\prime} \mathbf{G}^{-1} \mathbf{X}\right)=(p-1)\left(\frac{(n-3) p}{4(p-1)}\right)^{p} \\
\left\{\begin{array}{c}
\prod_{h=1}^{3}\left(1+\frac{g_{h}\left(p^{3}+8\right)}{(n-3) p^{2}}\right)+2\left(\frac{(p+2)(p-4)}{(n-3) p^{2}}\right)^{3} \prod_{h=1}^{3} g_{h}- \\
\left(\frac{(p+2)(p-4)}{(n-3) p^{2}}\right)^{2} \sum_{h, s, z} g_{h} g_{s}\left(1+\frac{g_{z}\left(p^{3}+8\right)}{(n-3) p^{2}}\right)
\end{array}\right\} \\
\left\{\begin{array}{c}
\operatorname{det}\left(\mathbf{X}^{\prime} \mathbf{G}^{-1} \mathbf{X}\right)=(p-1)\left(\frac{(n-3) p}{4(p-1)}\right)^{p} \\
\prod_{h=1}^{3}\left(1+\frac{g_{h}\left(p^{3}+8\right)}{(n-3) p^{2}}\right)^{3}+2\left(\frac{(p+2)(p-4)}{(n-3) p^{2}}\right)^{3} \prod_{h=1}^{3} g_{h}- \\
\left(\frac{(p+2)(p-4)}{(n-3) p^{2}}\right)_{h, s, z}^{2} g_{h} g_{s}\left(1+\frac{g_{z}\left(p^{3}+8\right)}{(n-3) p^{2}}\right)^{2}
\end{array}\right\} .
\end{gathered}
$$

Example 2.5. Let us consider the variance matrix of errors $\sigma^{2} \mathbf{G}$, where $\mathbf{G}^{-1}=\left[\begin{array}{cccc}\mathbf{I}_{6} & \mathbf{0}_{6} & \mathbf{0}_{6} & \mathbf{0}_{6} \\ \mathbf{0}_{6}^{\prime} & 5 & 0 & 0 \\ \mathbf{0}_{6}^{\prime} & 0 & 3 & 0 \\ \mathbf{0}_{6}^{\prime} & 0 & 0 & 7\end{array}\right]$. We determine a highly D-efficient design in the class $\mathbf{X} \in \Psi_{9 \times 4}(0,1)$. So, take the highly D-efficient spring balance weighing design

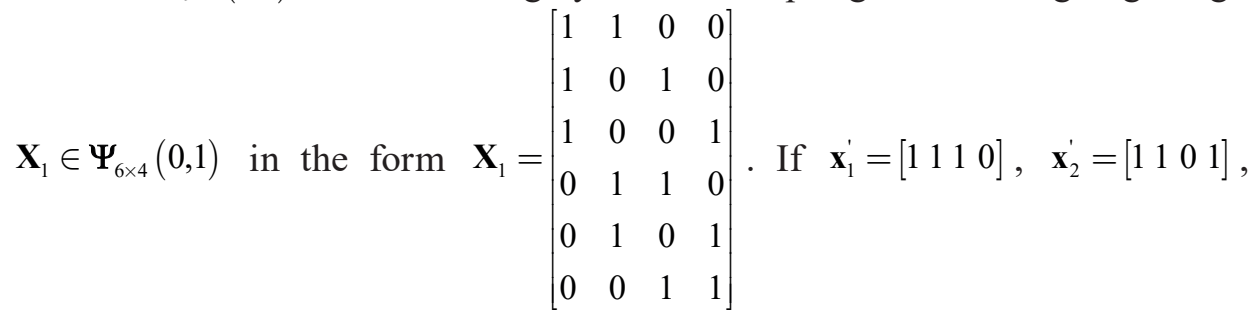

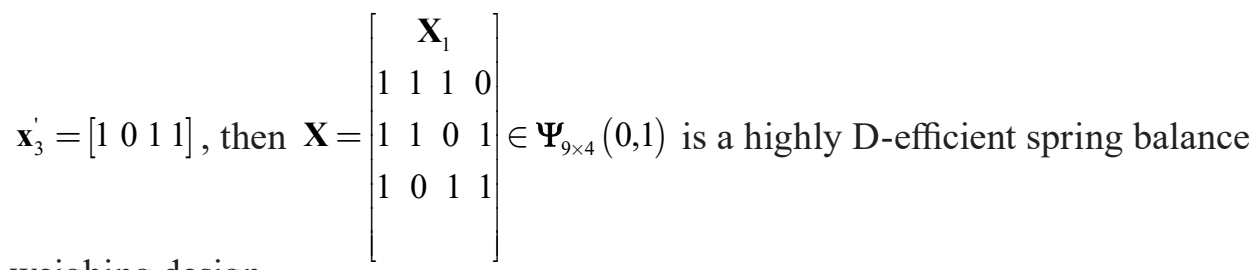
weighing design. 
Example 2.6. Let us consider the variance matrix of errors $\sigma^{2} \mathbf{G}$, where $\mathbf{G}^{-1}=\left[\begin{array}{cccc}\mathbf{I}_{10} & \mathbf{0}_{10} & \mathbf{0}_{10} & \mathbf{0}_{10} \\ \mathbf{0}_{10}^{\prime} & 5 & 0 & 0 \\ \mathbf{0}_{10}^{\prime} & 0 & 3 & 0 \\ \mathbf{0}_{10}^{\prime} & 0 & 0 & 7\end{array}\right]$. We determine a highly D-efficient design in the class $\mathbf{X} \in \Psi_{13 \times 6}(0,1)$. So, take the highly D-efficient spring balance weighing $\operatorname{design} \mathbf{X}_{1} \in \Psi_{10 \times 6}(0,1)$ in the form $\mathbf{X}_{1}=\left[\begin{array}{cccccc}1 & 1 & 0 & 0 & 1 & 0 \\ 1 & 1 & 0 & 0 & 0 & 1 \\ 1 & 0 & 1 & 1 & 0 & 0 \\ 1 & 0 & 1 & 0 & 0 & 1 \\ 1 & 0 & 0 & 1 & 1 & 0 \\ 0 & 1 & 1 & 1 & 0 & 0 \\ 0 & 1 & 1 & 0 & 1 & 0 \\ 0 & 1 & 0 & 1 & 0 & 1 \\ 0 & 0 & 1 & 0 & 1 & 1 \\ 0 & 0 & 0 & 1 & 1 & 1\end{array}\right]$. If $\mathbf{x}_{1}^{\prime}=\left[\begin{array}{lllll}1 & 1 & 1 & 1 & 0\end{array}\right]$,

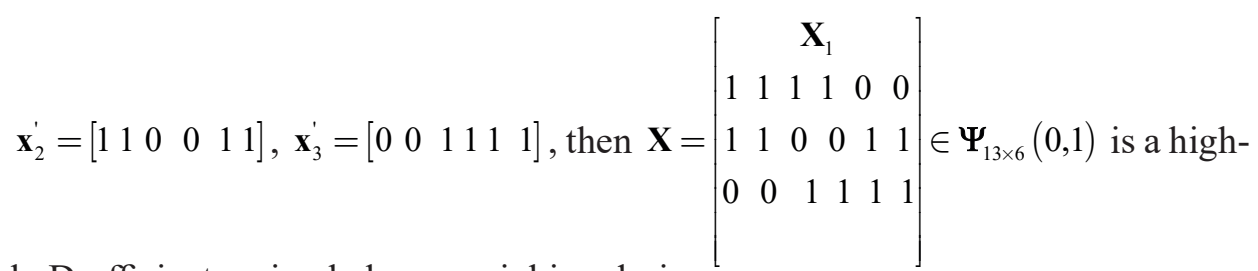
ly D-efficient spring balance weighing design.

\section{Conclusions}

Here, the theory and practice of the spring balance weighing design is presented. It is not possible to determine the D-optimal spring balance weighing design in any class $\boldsymbol{\Psi}_{n \times p}(0,1)$. Because of this, new construction methods of highly D-efficient designs in such classes are considered. The methods of determining highly D-efficient designs in classes in which D-optimal spring balance weighing designs have not been determined so far in the literature are presented in the examples. It is worth noting that in the highly D-efficient spring balance weighing design we are able to determine unknown measurements of objects with the minimum product of variances of their estimators. 
It is worth emphasising that spring balance weighing designs can be applied in all experiments in which the experimental factors are at two levels, see, for example, Ceranka and Graczyk (2014). Let us suppose that we study the real estate market and we are interested in the influence of the following factors: the prospect of further price increases in the local housing market, availability of loans for the purchase of apartments, the prospect of increasing VAT, fears related to the liquidation of the interest relief, the current price increase observed in the local market, availability of housing in the secondary market (each at two levels coded with 1 or 0 ). From the statistical point of view, we are interested in determining the influences of these factors using twenty different combinations. In the notation of weighing designs, we determine unknown measurements of $p=6$ objects in $n=13$ surveys, so we consider the class $\Psi_{13 \times 6}(0,1)$. The scheme of determination of the measurements, i.e. the design matrix, is given in the example 2.6. Possible applications of the discussed designs should be sought wherever the measurement results can be written as a linear combination of unknown object measures with coefficients equal to 0 or 1.

This paper was presented at the conference MSA 2019 which financed its publication. Organisation of the international conference "Multivariate Statistical Analysis 2019" (MSA 2019) was supported by resources for the popularisation of scientific activities of the Minister of Science and Higher Education in the framework of agreement No 712/PDUN/202019.

\section{References}

Banerjee K.S. (1975), Weighing Designs for Chemistry, Medicine, Economics, Operation Research, Statistics, Marcell Dekker Inc., New York.

Bulutoglu D.A., Ryan K.J. (2009), D-optimal and near D-optimal $2^{\mathrm{k}}$ fractional factorial designs of Resolution V, "Journal of Statistical Planning and Inference", no. 139, pp. 16-22.

Ceranka B., Graczyk M. (2010), Some construction of optimum weighing designs, "Acta Universitatis Lodziensis. Folia Oeconomica", no. 235, pp. 235-239.

Ceranka B., Graczyk M. (2012), Notes on the optimum chemical balance weighing designs, "Acta Universitatis Lodziensis. Folia Oeconomica", no. 269, pp. 91-101.

Ceranka B., Graczyk M. (2014), On certain A-optimal biased spring balance weighing designs, "Statistics in Transition. New Series", Spring, vol. 15(2), pp. 317-326.

Ceranka B., Graczyk M. (2018), Highly D-efficient designs for even number of objects, "Revstat-Statistical Journal", no. 6, pp. 475-486.

Ceranka B., Graczyk M. (2019), A highly D-efficient spring balance weighing designs for an even number of objects, "Acta Universitatis Lodziensis. Folia Oeconomica", no. 344, pp. 17-27.

Ceranka B., Katulska K. (1987a), Zastosowanie optymalnych sprężynowych układów wagowych, [in:] Siedemnaste Colloquium Metodologiczne z Agro-Biometrii, PAN, Warszawa, pp. 98-108.

Ceranka B., Katulska K. (1987b), Zastosowanie teorii sprężynowych układów wagowych do analizy doświadczeń z mieszankami, "Listy Biometryczne", no. XXIV, pp. 17-26.

Ceranka B., Katulska K. (1989), Application of the biased spring balance weighing theory to estimation of differences of line effects for legume content, "Biometrical Journal", no. 31, pp. 103-110. 
Gail Z., Kiefer J. (1982), Construction methods for D-optimum weighing designs when $n \equiv 3$ mod 4, "The Annals of Statistics", no. 10, pp. 502-510.

Gawande B.N., Patkar A.Y. (1999), Application of factorial design for optimization of Cyclodextrin Glycosyltransferase production from Klebsiella Pneumoniae AS-22, "Biotechnology and Bioengineering”, no. 64, pp. 168-173.

Graczyk M. (2013), Some applications on weighing designs, "Biometrical Letters", vol. 50(1), pp. $15-26$.

Jacroux M., Notz W. (1983), On the Optimality of Spring Balance Weighing Designs, "The Annals of Statistics", no. 11, pp. 970-978.

Katulska K., Smaga Ł. (2010), On some construction of D-optimal chemical balance weighing designs, "Colloquium Biometricum", no. 40, pp. 37-45.

Koukouvinos Ch. (1996), Linear models and D-optimal designs for $n \equiv 2 \bmod 4$, "Statistics and Probability Letters", no. 26, pp. 329-332.

Raghavarao D. (1971), Constructions and Combinatorial Problems in Designs of Experiments, John Wiley Inc., New York.

Shah K.R., Sinh B.K. (1989), Theory of Optimal Designs, Springer Verlag, Berlin.

\section{Uwagi o wysoce D-efektywnych sprężynowych układach wagowych}

Streszczenie: W artykule rozważamy nowe metody konstrukcji wysoce D-efektywnych sprężynowych układów wagowych w klasach, w których nie istnieje układ D-optymalny. Podajemy warunki wyznaczające relacje pomiędzy parametrami tych układów oraz przykłady konstrukcji.

Słowa kluczowe: sprężynowy układ wagowy, układ D-efektywny

JEL: C02, C18, C90

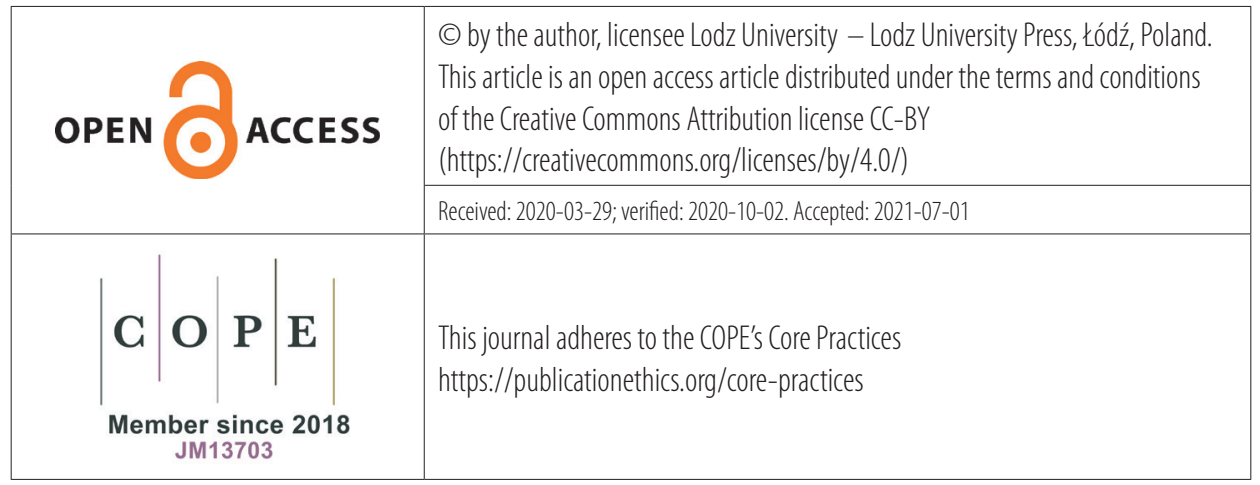

